\title{
Predictability on finite horizon for processes with exponential decrease of energy on higher frequencies
}

\author{
Nikolai Dokuchaev \\ Department of Mathematics, Trent University, Ontario, Canada \\ Revised version: May 7th, 2009; the first version: November 17, 2008
}

\begin{abstract}
The paper presents sufficient conditions of predictability for continuous time processes in deterministic setting. We found that processes with exponential decay on energy for higher frequencies are predictable in some weak sense on some finite time horizon defined by the rate of decay. Moreover, this predictability can be achieved uniformly over classes of processes. Some explicit formulas for predictors are suggested.
\end{abstract} Key words: nonparametric methods, spectral analysis, forecasting AMS 2000 classification : 60G25, 93E10, 42B30.

\section{Introduction}

We study pathwise predictability of continuous time processes in deterministic setting and in the framework of the frequency analysis. It is well known that certain restrictions on frequency distribution can ensure additional opportunities for prediction and interpolation of the processes. The classical result is Nyquist-Shannon-Kotelnikov interpolation theorem for the band-limited processes. There are related predictability results; see, e.g., Wainstein and Zubakov (1962), Beutler (1966), Brown(1969), Slepian (1978), Knab (1981), Papoulis (1985), Marvasti (1986), Vaidyanathan (1987), Lyman et al (2000, 2001). These works considered predictability of single processes, and the crucial assumption was that the processes are band-limited; the predictors were non-robust with respect to small noise in high frequencies; see, e.g., the discussion in Chapter 17 from Higgins (1996).

We study some special weak predictability of continuous time processes. Instead of predictability of the original processes, we study predictability of sets of anticausal convolution integrals for a wide enough classes of kernels. This version of predictability was 
introduced in Dokuchaev (2008) for band-limited processes; it allowed to establish uniform predictability in this weakened sense over classes of band-limited and high-frequency processes. In the present paper, we established some predicability for continuous time processes with exponential decay of energy on the higher frequencies. It allows to consider processes that are not band-limited. More precisely, we obtain a sufficient condition of uniform weak predictability on prediction horizon $T$ over some classes of processes with exponential decay of energy on higher frequencies $\omega \rightarrow \pm \infty$, when the energy is decreasing faster than $e^{-T|\omega|}$. An alternative formulation of this condition in time domain is also given, The predictors are obtained explicitly in the frequency domain via their transfer function. These predictors are defined entirely by the kernel of the convolution integral and their choice is independent from the characteristics of the particular input processes.

\section{Problem setting and definitions}

Let $x(t)$ be a currently observable continuous time process, $t \in \mathbf{R}$. The goal is to estimate, at a current time $t$, the values $y(t)=\int_{t}^{t+T} k(t-s) x(s) d s$, where $k(\cdot)$ is a given kernel, and $T>0$ is a given prediction horizon. At any time $t$, the predictors use historical values of the observable process $\left.x(s)\right|_{s \leq t}$.

We consider only linear predictors in the form $\widehat{y}(t)=\int_{-\infty}^{t} \widehat{k}(t-s) x(s) d s$, where $\widehat{k}(\cdot)$ is a kernel that has to be found. We will call $\widehat{k}$ a predictor or predicting kernel.

Let us describe admissible classes of $k$ and $\widehat{k}$.

Let $\mathbf{R}^{+} \triangleq[0,+\infty), \mathbf{C}^{+\triangleq} \triangleq\{z \in \mathbf{C}: \operatorname{Re} z>0\}, i=\sqrt{-1}$.

For $x \in L_{2}(\mathbf{R}) \cup L_{1}(\mathbf{R})$, we denote by $X=\mathcal{F} x$ the function defined on $i \mathbf{R}$ as the Fourier transform of $x$;

$$
X(i \omega)=(\mathcal{F} x)(i \omega)=\int_{-\infty}^{\infty} e^{-i \omega t} x(t) d t, \quad \omega \in \mathbf{R} .
$$

If $x \in L_{2}(\mathbf{R})$, then $X$ is defined as an element of $L_{2}(\mathbf{R})$ (more precisely, $X(i \cdot) \in L_{2}(\mathbf{R})$ ).

For $x(\cdot) \in L_{2}(\mathbf{R})$ such that $x(t)=0$ for $t<0$, we denote by $\mathcal{L} x$ the Laplace transform

$$
X(p)=(\mathcal{L} x)(p) \triangleq \int_{0}^{\infty} e^{-p t} x(t) d t, \quad p \in \mathbf{C}^{+} .
$$

Let $H^{r}$ be the Hardy space of holomorphic on $\mathbf{C}^{+}$functions $h(p)$ with finite norm $\|h\|_{H^{r}}=\sup _{s>0}\|h(s+i \omega)\|_{L_{r}(\mathbf{R})}, r \in[1,+\infty]$ (see, e.g., Duren (1970)).

Definition 1 For $T>0$, we denote by $\mathcal{K}(T)$ the set of functions $k: \mathbf{R} \rightarrow \mathbf{R}$ such that $k(t)=0$ for $t \notin[-T, 0]$ and such that $k \in L_{\infty}(\mathbf{R})$. 
Definition 2 Let $\widehat{\mathcal{K}}$ be the class of functions $\widehat{k}: \mathbf{R} \rightarrow \mathbf{R}$ such that $\widehat{k}(t)=0$ for $t<0$ and such that $K(\cdot)=\mathcal{L} \widehat{k} \in H^{2} \cap H^{\infty}$.

We consider below $k \in \mathcal{K}(T)$ and $\widehat{k} \in \widehat{\mathcal{K}}$.

Definition 3 Let $\overline{\mathcal{X}}$ be a class of processes $x(\cdot)$ from $L_{2}(\mathbf{R}) \cup L_{1}(\mathbf{R})$. Let $r \in[1,+\infty]$.

(i) We say that the class $\overline{\mathcal{X}}$ is $L_{r}$-predictable in the weak sense with the prediction horizon $T$ if, for any $k(\cdot) \in \mathcal{K}(T)$, there exists a sequence $\left\{\widehat{k}_{m}(\cdot)\right\}_{m=1}^{+\infty}=$ $\left\{\widehat{k}_{m}(\cdot, \overline{\mathcal{X}}, k)\right\}_{m=1}^{+\infty} \subset \widehat{\mathcal{K}}$ such that

$$
\left\|y-\widehat{y}_{m}\right\|_{L_{r}(\mathbf{R})} \rightarrow 0 \quad \text { as } \quad m \rightarrow+\infty \quad \forall x \in \mathcal{X}
$$

where

$$
y(t) \triangleq \int_{t}^{t+T} k(t-s) x(s) d s, \quad \widehat{y}_{m}(t) \triangleq \int_{-\infty}^{t} \widehat{k}_{m}(t-s) x(s) d s .
$$

The process $\widehat{y}_{m}(t)$ is the prediction of the process $y(t)$ which describes depends on the future values of process $\left.x(s)\right|_{s \in[t, t+T]}$.

(ii) Let the set $\mathcal{F}(\overline{\mathcal{X}}) \triangleq\{X=\mathcal{F} x, \quad x \in \overline{\mathcal{X}}\}$ be provided with a norm $\|\cdot\|$. We say that the class $\overline{\mathcal{X}}$ is $L_{r}$-predictable in the weak sense with the prediction horizon $T$ uniformly with respect to the norm $\|\cdot\|$, if, for any $k(\cdot) \in \mathcal{K}(T)$, there exists a sequence $\left\{\widehat{k}_{m}(\cdot)\right\}=\left\{\widehat{k}_{m}(\cdot, \mathcal{X}, k,\|\cdot\|, \varepsilon)\right\} \subset \widehat{\mathcal{K}}$ such that

$$
\|y-\widehat{y}\|_{L_{r}(\mathbf{R})} \rightarrow 0 \quad \text { uniformly in } \quad\{x \in \overline{\mathcal{X}}:\|X\| \leq 1\} .
$$

Here $y(\cdot)$ and $\widehat{y}_{m}(\cdot)$ are the same as above.

\section{The main result}

For $q \in\{1,2\}$, let $\mathcal{X}(q)=\mathcal{X}(q, T)$ be the set of processes $x(\cdot) \in L_{2}(\mathbf{R}) \cup L_{1}(\mathbf{R})$ such that

$$
\int_{-\infty}^{+\infty} e^{q T|\omega|}|X(i \omega)|^{q} d \omega<+\infty, \quad X(i \omega)=\mathcal{F} x
$$

For $\Omega>0$, set $D(\Omega) \triangleq \mathbf{R} \backslash(-\Omega, \Omega)$.

Clearly, if $x(\cdot) \in \mathcal{X}(q, T)$, then

$$
\int_{D(\Omega)} e^{q T|\omega|}|X(i \omega)|^{q} d \omega \rightarrow 0 \quad \text { as } \quad \Omega \rightarrow+\infty .
$$

It can be seen also that, for any $T>0$, the class $\mathcal{X}(q, T)$ includes all band-limited processes $x$ such that $X(i \omega)=\mathcal{F} x \in L_{q}(\mathbf{R}), q \in\{1,2\}$. 
Theorem 1 Let $q \in\{1,2\}$. Set $r=r(q)=q(q-1)^{-1}$ (in particular, $r=\infty$ for $q=1$ and $r=2$ for $q=2$ ).

(i) The class $\mathcal{X}(q, T)$ is $L_{r}$-predictable in the weak sense with the prediction horizon $T$.

(ii) Let $\mathcal{U}(q)=\mathcal{U}(q, T)$ be a class of processes $x(\cdot) \in \mathcal{X}(q, T)$ such that

$$
\int_{D(\Omega)} e^{q T|\omega|}|X(i \omega)|^{q} d \omega \rightarrow 0 \quad \text { as } \quad \Omega \rightarrow+\infty \quad \text { uniformly on } \quad x(\cdot) \in \mathcal{U}(q) .
$$

Then this class $\mathcal{U}(q, T)$ is $L_{r}$-predictable in the weak sense with the prediction horizon $T$ uniformly with respect to the norm $\|\cdot\|_{L_{q}(\mathbf{R})}$.

Some alternative descriptions and examples of sets $\mathcal{U}(q, T)$ are given below.

The question arises how to find the predicting kernels. In the proof of Theorem 1, a possible choice of the kernels is given explicitly in the frequency domain, i.e., via the transfer functions.

Remark 1 In Dokuchaev (2008), similar weak predictability with infinite horizon was introduced and established for models where an ideal low-pass filter exists; the predictors used in this paper were different from the ones presented below. Theorem 1 allows to extend this weak predictability on the case when the filters are not ideal but allow exponentially decay of energy on higher frequencies.

Remark 2 The case when processes $k(\cdot) \in L_{2}(R) \backslash L_{\infty}(\mathbf{R})$ can be also covered. In this case, we have to require that $x \in L_{2}(\mathbf{R})$.

\section{On possibility of extrapolation}

The weak predictability introduced in Theorem 1 does not ensure extrapolation of the processes from $\mathcal{U}(q, T)$ in the classical sense; even approximate extrapolation is not guaranteed. Let us explain why. Let $q=2$ and $T>0$ be given. Assume that the values of the process $x(t) \in \mathcal{U}(2, T)$ are known for $t \leq \tau$. Consider a sequence of kernels $\left\{K_{m}\right\}_{m=1}^{+\infty} \subset \mathcal{K}(T)$ that forms a orthonormal basis in the Hilbert space $L_{2}(-T, 0)$ (for instance, one may take Fourier series). For any $\tau$, the function $\left.x(t)\right|_{[\tau, \tau+T]} \in L_{2}(\tau, \tau+T)$ can be represented as a series $x(t)=\sum_{m=1}^{\infty} f_{m} K_{m}(\tau+T-t)$, where $f_{m}=\int_{\tau}^{\tau+T} K_{m}(\tau+T-s) x(s) d s$ are the corresponding Fourier coefficients (the series converges in $L_{2}(\tau, \tau+T)$ ). Theorem 1 ensures that, for any $\varepsilon>0$, there exists a predictor such that the values of $f_{m}$ can be predicted at time $t$ with the error less or equal than $\varepsilon$ for all $m$. Unfortunately, it does not help to predict the summa of infinite series, even if this $\varepsilon$ is small. 


\section{On predicability and causality}

It may appears that Theorem 1 contradicts to the obvious fact that a general process cannot be predicted in any sense. For instance, let $x_{1}(t)$ and $x_{2}(t)$ be two processes such that $x_{1}(t)=x_{2}(t)=0$ for $t \leq 0$ and such that $x_{1}(t) \neq x_{2}(t)$ for $t>0$. Clearly, it is not possible to say which process we observe at time $t=0$ using the values for $t \leq 0$. (Some discussion and examples related to the predictability can be found in Chapter 17 from Higgins (1996)). However, it does not contradict to Theorem 1, For instance, let $x_{k}(t)=(-1)^{k} t e^{-t}$ for $t>0$; it is easy to verify that the Fourier transforms of the processes $x_{k}(\cdot)$ do not belong to $\mathcal{X}(q), q=1,2$. It reflects the lack of causality for these process: the values for $t>0$ cannot be regarded as continuation of some development started before $t=0$. In contrast, periodic, almost-periodic, and band-limited processes have causality property and therefore can be predicted.

Theorem 1 says that the predictability can be ensured for some processes other than periodic, almost-periodic, or band-limited. In particular, there is some causality for all processes covered by this theorem, i.e., some signs of future development are presented in the current time. Therefore, Theorem 1 can be interpreted as a new sufficient frequency condition of causality for processes that are not periodic, almost-periodic, or band-limited. In some cases, this causality makes possible predictability on some fixed finite horizon only. In particular, these conditions are more restrictive for longer horizon.

\section{Sufficient conditions of predictability in time domain}

In Theorem 1, conditions of predictability are formulated in frequency domain. It can be useful to add some sufficient conditions in time domain.

For $C>0$, consider a class $\mathcal{M}(C)$ of processes $x(t) \in C^{\infty}(\mathbf{R})$ such that there exists $M>0$ such that

$$
\begin{aligned}
& \left\|\frac{d^{k} x}{d t^{k}}(\cdot)\right\|_{L_{2}(\mathbf{R})}^{2} \leq C^{k} M, \quad k=0,2,4,6, \ldots \\
& \frac{1}{2}\left(\left\|\frac{d^{k-1} x}{d t^{k-1}}(\cdot)\right\|_{L_{2}(\mathbf{R})}^{2}+\left\|\frac{d^{k+1} x}{d t^{k+1}}(\cdot)\right\|_{L_{2}(\mathbf{R})}^{2}\right) \leq C^{k} M, \quad k=1,3,5,7, \ldots \quad \forall x(\cdot) \in \mathcal{M},
\end{aligned}
$$

Proposition 1 For any $T>0$ and $C>0, \mathcal{M}(C) \subset \mathcal{X}(2, T)$. In particular, for any $T>0$ and $C>0$, the class $\mathcal{M}(C)$ is predictable in the weak sense with the prediction horizon $T$. 
For $C>0$, consider a class $\mathcal{N}(C)$ of processes $x(t) \in C^{\infty}(\mathbf{R})$ such that there exists $M>0$ such that

$$
\begin{gathered}
\left\|\frac{d^{k} x}{d t^{k}}(\cdot)\right\|_{L_{2}(\mathbf{R})}^{2} \leq k ! C^{-k} M, \quad k=0,2,4,6, \ldots \\
\frac{1}{2}\left(\left\|\frac{d^{k-1} x}{d t^{k-1}}(\cdot)\right\|_{L_{2}(\mathbf{R})}^{2}+\left\|\frac{d^{k+1} x}{d t^{k+1}}(\cdot)\right\|_{L_{2}(\mathbf{R})}^{2}\right) \leq k ! C^{-k} M, \quad k=1,3,5,7, \ldots . \quad \forall x(\cdot) \in \mathcal{N}(C) .
\end{gathered}
$$

Proposition 2 For any $C>2 T, \mathcal{N}(C) \subset \mathcal{X}(2, T)$. In particular, the class $\mathcal{N}(C)$ is predictable in the weak sense with the prediction horizon $T<C / 2$.

\section{Example: outputs of Gaussian filters}

Let $\bar{C}>0$ and $\bar{\omega}>0$ be given. Consider a class of processes $\mathcal{Z}(\bar{C}, \bar{\omega})=\{z(\cdot)\}$ such that the Fourier transform $Z(i \omega)=\mathcal{F} z$ is defined in the class $L_{2}(\mathbf{R}) \cup L_{\infty}(\mathbf{R})$ and $|X(1 \omega)| \leq \bar{C}$ for all $\omega$ such that $|\omega|>\bar{\omega}$, where $z \in \mathcal{Z}$.

Let $c_{1}>0$ and $v_{1}>0$ be given. Consider a set $\mathcal{G}=\mathcal{G}\left(c_{1}, v_{2}\right)$ of Gaussian filters with kernels $k_{\mathcal{G}}(t)=c \exp \left(-\frac{t^{2}}{v}\right)$ such that $v \geq v_{1}$ and $|c| \leq c_{1}$.

Let $\mathcal{V}_{\mathcal{G}}=\mathcal{V}_{G}\left(\bar{C}, \bar{c}, c_{1}, v_{1}\right)$ be the set of processes $x$ such that $x$ is a convolution of $z$ with a kernel $k_{\mathcal{G}}$, where $k_{\mathcal{G}} \in \Gamma\left(c_{1}, v_{1}\right), z \in \mathcal{Z}(\bar{C}, \bar{\omega})$, i.e.,

$$
x(t)=\int_{-\infty}^{\infty} k_{\mathcal{G}}(t-s) z(s) d s .
$$

Note that generalized functions $v \in C(\mathbf{R})^{*}$ are allowed to be elements of $\mathcal{Z}$ (for instance, we include delta functions). In that case, $\mathcal{F} z$ is still well defined, and the corresponding process $x=\mathcal{F}^{-1} X(i \omega)$ is well defined in $L_{2}(\mathbf{R})$, where $X(i \omega)=K_{\mathcal{G}}(i \omega) Z(i \omega), K_{\mathcal{G}}(i \omega)=$ $\mathcal{F} k_{\mathcal{G}}$.

Proposition 3 (i) $\mathcal{V}_{\mathcal{G}} \subset \mathcal{X}(1) \cap \mathcal{X}(2)$; (ii) For $q=1$, 2, for any $T>0$, the class $\mathcal{V}_{\mathcal{G}}$ is predicted in the weak sense with the prediction horizon $T$ uniformly with respect to the norm $\|\cdot\|_{L_{q}(\mathbf{R})}$.

For example, consider processes

$$
x(t)=\sum_{m=1}^{N} c_{m} \exp \left(-\frac{\left(t-a_{m}\right)^{2}}{v_{m}}\right) .
$$

for some constants $N>0, c_{m}, a_{m}$, and $v_{m}>0$. By Proposition 3 , these processes belong to $\mathcal{X}(1) \cap \mathcal{X}(2)$, and that any set of these processes such that

$$
\left\{N \leq C_{1},\left|c_{m}\right| \leq C_{2}, v_{m} \geq C_{3},\left|a_{m}\right| \leq C_{4}\right\}
$$


forms a class $\mathcal{U}(q)$ with the properties required in Theorem 1 , for any given set of positive $C_{1}, C_{2}, C_{3}, C_{4}$. Therefore, these processes can be predicted and uniformly predicted in the weak sense of Theorem 1. In particular, it is possible to predict for any $T>0$ the values

$\int_{t}^{t+T} k(t-s) x(s) d s$ using the values for $\theta<t$ and the predictors defined in the proof of Theorem 1. If $a_{m}>0$ and $v_{m}^{-1}$ are large enough, then the processes have sharp peaks in $t>0$, and the values of $x(\theta)$ are small for $\theta \leq 0$, and the impact of the choice of $N, c_{k}$, and $a_{k}$ on $\left.y(\cdot)\right|_{(-\infty, 0]}$ is small. However, this impact still exists, ant it makes the prediction at time $t=0$ possible.

\section{Special case: extrapolation of the still snapshot of the temperature}

We have regarded $x(t)$ as processes in time with the time variable $t$. It is the most natural model for prediction. However, there are other models where Theorem 1 can be applied. For instance, consider the problem of measurement of the temperature on the one-dimensional rod. We consider the still snapshot of the temperature rather than the dynamics of the process of heat propagation. Let $x(t)$ be the temperature at the point with the coordinate $t \in \mathbf{R}$. Let us assume that the temperature is given as (5.1) with $c_{m}>0$; this case corresponds to the model when the heat was originated from $N$ point sources that were applied at the points $t=a_{m}>0$ at past times defined by $v_{m}$. We assume that $N, a_{m}, c_{m}$, and $v_{m}$ are unknown and non-observable. Assume that the temperature can be measured in the points $t \in(-\infty, 0]$ only. The problem is to estimate integrals $\int_{0}^{T} k(-s) x(s) d s$ using the observations at $t \in[0,+\infty)$ only, with $T>0$; in fact, it is a relaxed version of the extrapolation problem. Theorem 1 gives the solution, and the "predictor" from the proof can be used.

\section{Appendix: Proofs}

The proofs below are very straightforward and do not use the advanced theory of $H^{p_{-}}$ spaces; the existence of required predictors is proved by presenting explicit transfer functions of the predictors with desired properties.

Let $k(\cdot) \in \mathcal{K}(T)$ and $K(i \omega)=\mathcal{F} k$. We assume here and below that $\omega \in \mathbf{R}$.

For $\gamma \in \mathbf{R}, \gamma>0$, set

$$
g(p) \triangleq T \frac{\gamma-p}{\gamma+p} p, \quad h(p) \triangleq g(p)-T p, \quad V(p) \triangleq e^{h(p)}
$$


Lemma 1 (i) $V(p) \in H^{\infty}$ and $\widehat{K}(i \omega)=V(i \omega) K(i \omega)$ can be extended on $\mathbf{C}^{+}$as function $\widehat{K}(p) \in H^{2} \cap H^{\infty}$.

(ii) $|V(i \omega)|=\exp \left(\frac{2 T \gamma \omega^{2}}{\gamma^{2}+\omega^{2}}\right)$.

(iii) $\sup _{\gamma>0}|V(i \omega)| \leq e^{T|\omega|}$.

(iv) $V(i \omega) \rightarrow 1$ as $\gamma \rightarrow+\infty$ for all $\omega \in \mathbf{R}$.

(v) For any $\varepsilon>0$ and any $\Omega>0$, there exists $\gamma>0$ such that $|V(i \omega)-1| \leq \varepsilon$ for all $\omega \in[-\Omega, \Omega]$.

Proof of Lemma 11. Set $Q(i \omega)=e^{-i \omega T} K(i \omega)$, i.e., $K(i \omega)=e^{i \omega T} K(i \omega)$. Clearly,

$$
Q(i \omega)=e^{-i \omega T} \int_{-T}^{0} e^{-i \omega t} k(t) d t=\int_{0}^{T} e^{-i \omega(T-\theta)} k(-\theta) d \theta=\int_{0}^{T} e^{-i \omega \tau} k(\tau-T) d \tau
$$

It follows that $Q(i \omega)$ can be extended on $\mathbf{C}^{+}$as function $Q(p) \in H^{2} \cap H^{\infty}$.

Further, $V(p)=e^{-T p} e^{g(p)}$ and

$$
g(p)=T \frac{\gamma-p}{\gamma+p} p=T \frac{-\gamma-p+2 \gamma}{\gamma+p} p=-T p+T \frac{2 \gamma p}{\gamma+p} .
$$

It follows that $e^{g(p)} \in H^{\infty}$. Hence $\widehat{K}(i \omega)=V(i \omega) K(i \omega)=Q(i \omega) e^{g(i \omega)}$ can be extended on $\mathbf{C}^{+}$as function $\widehat{K}(p) \in H^{2} \cap H^{\infty}$. Then statement (i) follows.

Further,

$$
g(i \omega)=T \frac{\gamma-i \omega}{\gamma+i \omega} i \omega=T \frac{(\gamma-i \omega)^{2}}{\gamma^{2}+\omega^{2}} i \omega=T \frac{\gamma^{2}-2 \gamma i \omega-\omega^{2}}{\gamma^{2}+\omega^{2}} i \omega
$$

Then

$$
\operatorname{Re} h(i \omega)=\operatorname{Re} g(i \omega)=\frac{2 T \gamma \omega^{2}}{\gamma^{2}+\omega^{2}}
$$

Then statement (ii) follows.

Let us find the maximum of $\operatorname{Re} h(p)=\operatorname{Re} h(p, \gamma)$ in $\gamma \geq 0$. It suffices to find $\gamma$ such that $\frac{\partial}{\partial \gamma} \operatorname{Re} h(i \omega)=0$, i.e., such that

$$
\frac{\partial}{\partial \gamma}\left(\frac{2 T \gamma \omega^{2}}{\gamma^{2}+\omega^{2}}\right)=\frac{2 T \omega^{2}\left(\gamma^{2}+\omega^{2}\right)-4 T \omega^{2} \gamma^{2}}{\left(\gamma^{2}+\omega^{2}\right)^{2}}=2 T \omega^{2} \frac{\gamma^{2}+\omega^{2}-2 \gamma^{2}}{\left(\gamma^{2}+\omega^{2}\right)^{2}}=0
$$

It is easy to see that (6.1) holds for $\gamma=|\omega|$. For this $\gamma=|\omega|$, we have that

$$
\operatorname{Re} h(i \omega)=\frac{2 T \gamma \omega^{2}}{\gamma^{2}+\omega^{2}}=\frac{2 T|\omega| \omega^{2}}{2 \omega^{2}}=T|\omega| .
$$

Hence (iii) follows. 
We have that

$$
h(p)=h(p, \gamma)=-T p \frac{2 p}{\gamma+p}
$$

Hence $h(i \omega, \gamma) \rightarrow 0$ as $\gamma \rightarrow+\infty$ for any $w \in \mathbf{R}$. Then statement (iv) follows.

Further, it follows from continuity of the exponent function that there exists a function $\psi(\cdot):(0,+\infty) \rightarrow(0,+\infty)$ such that if $|h(i \omega)| \leq \psi(\varepsilon)$ then $|V(i \omega)-1|<\varepsilon$. Let an arbitrarily small $\varepsilon>0$ and an arbitrarily large $\Omega>0$ be given. Take $\gamma=\gamma(\varepsilon) \geq$ $2 T \Omega^{2} \psi(\varepsilon)^{-1}$, then

$$
|h(i \omega)|^{2}=\frac{4 T^{2} \omega^{4}}{\gamma(\varepsilon)^{2}+\omega^{2}} \leq \psi(\varepsilon)^{2} \quad \forall \omega \in[-\Omega, \Omega]
$$

i.e.,

$$
|V(i \omega)-1| \leq \varepsilon \quad \forall \omega \in[-\Omega, \Omega]
$$

Then statement (v) follows. This completes the proof of Lemma 1

Proof of Theorem 1] It suffices to present a set of predicting kernels $\widehat{k}$ with desired properties. Let $V(\cdot)=V(\gamma, \cdot)$ be as defined in Lemma 1, Set $\widehat{K}(i \omega) \triangleq V(i \omega) K(i \omega)$. Let the predicting kernels be defined as $\widehat{k}(\cdot)=\widehat{k}(\cdot, \gamma(\varepsilon))=\mathcal{F}^{-1} \widehat{K}(i \omega)$.

For $x(\cdot) \in L_{q}(\mathbf{R})$, let $X(i \omega) \triangleq \mathcal{F} x, Y(i \omega) \triangleq \mathcal{F} y=K(i \omega) X(i \omega)$. Set $\widehat{Y}(i \omega) \triangleq$ $\widehat{K}(i \omega) X(i \omega)=V(i \omega) Y(i \omega)$ and $\widehat{y}=\mathcal{F}^{-1} \widehat{Y}$.

Let us prove (i). Since $K(i \omega) \in L_{\infty}(\mathbf{R})$ and $X(i \omega) \in L_{q}(\mathbf{R})$, we have that $Y(i \omega)=$ $K(i \omega) X(i \omega) \in L_{q}(\mathbf{R})$ and $\widehat{Y} \in L_{q}(\mathbf{R})$. By Lemma 1(iv), it follows that

$$
\widehat{Y}(i \omega) \rightarrow Y(i \omega) \text { for a.e. } \omega \in \mathbf{R} \quad \text { as } \quad \gamma \rightarrow+\infty .
$$

We have that $e^{T|\omega|} X \in L_{q}(\mathbf{R}), K(i \omega) \in L_{\infty}(\mathbf{R})$ and

$$
\begin{aligned}
& |\widehat{K}(i \omega)-K(i \omega)| \leq|V(i \omega)-1||K(i \omega)| \leq 2 e^{T|\omega|}|K(i \omega)|, \quad \omega \in D, \\
& |\widehat{Y}(i \omega)-Y(i \omega)| \leq 2 e^{T|\omega|}|K(i \omega)||X(i \omega)|, \quad \omega \in D .
\end{aligned}
$$

By (6.2), (6.4), and by Lebesque Dominance Theorem, it follows that

$$
\|\widehat{Y}-Y\|_{L_{q}(\mathbf{R})} \rightarrow 0, \quad \text { i.e., } \quad\|\widehat{y}-y\|_{L_{r}(\mathbf{R})} \rightarrow 0 \quad \omega \in \mathbf{R} \quad \text { as } \quad \gamma \rightarrow+\infty .
$$

Let us prove (ii). Let $\varepsilon>0$ be given, and let $\Omega(\varepsilon) \triangleq \varepsilon^{-1}$. By Lemma 1(v), there exists $\gamma=\gamma(\varepsilon)>0$ such that $|V(i \omega)-1|^{q} \leq \varepsilon$ for all $\omega \in[-\Omega(\varepsilon), \Omega(\varepsilon)]$. For this $\gamma=\gamma(\varepsilon)$, we have

$$
\|\widehat{Y}(i \omega)-Y(i \omega)\|_{L_{q}(\mathbf{R})}^{q}
$$




$$
\begin{aligned}
& \leq \int_{-\Omega(\varepsilon)}^{\Omega(\varepsilon)}|V(i \omega)-1|^{q}|K(i \omega)|^{q}|X(i \omega)|^{q} d \omega+\int_{D(\Omega(\varepsilon))}|V(i \omega)-1|^{q}|K(i \omega)|^{q}|X(i \omega)|^{q} d \omega \\
& \leq\|K(i \omega)\|_{L_{\infty}(\mathbf{R})}^{q}\left(\varepsilon^{q}\|X(i \omega)\|_{L_{q}(\mathbf{R})}^{q}+2 \int_{D(\Omega(\varepsilon))} e^{q T|\omega|}|X(i \omega)|^{q} d \omega\right),
\end{aligned}
$$

Take $\varepsilon \rightarrow 0$. By (6.6) , it follows that $\|\widehat{Y}(i \omega)-Y(i \omega)\|_{L_{q}(\mathbf{R})}^{q} \rightarrow 0$ and $\|\widehat{y}-y\|_{L_{r}(\mathbf{R})} \rightarrow 0$ uniformly over $\mathcal{U}(q) \cap\left\{x(\cdot):\|X(i \omega)\|_{L_{q}(\mathbf{R})} \leq 1\right\}$.

By (6.5), (6.6), it follows that the predicting kernels $\widehat{k}(\cdot)=\widehat{k}(\cdot, \gamma(\varepsilon))=\mathcal{F}^{-1} \widehat{K}(i \omega)$ are such as required. This completes the proof of Theorem 1 .

Proof of Proposition 1, Let $x(\cdot) \in \mathcal{M}(C)$. Since $\exp (2|\omega| T)=\sum_{k=0}^{\infty} \frac{(2|\omega| T)^{k}}{k !}$ and $|\omega|^{k} \leq\left(|\omega|^{k-1}+|\omega|^{k+1}\right) / 2, k \geq 1$, we have that

$$
\int_{-\infty}^{+\infty} e^{2|\omega| T}|X(i \omega)|^{2} d \omega=\sum_{k=0}^{\infty} \frac{(2 T)^{k}}{k !} \int_{-\infty}^{+\infty}|\omega|^{k}|X(i \omega)|^{2} d \omega \leq 2 \pi M \sum_{k=0}^{\infty} \frac{(2 T C)^{k}}{k !}<+\infty .(6.7)
$$

It follows that $x(\cdot) \in \mathcal{X}(2, T)$ for all $T>0$. By Theorem 1(i), the required predictability holds.

Proof of Proposition 2, Let $x(\cdot) \in \mathcal{N}(C)$. Similarly to (6.7), we obtain that

$$
\int_{-\infty}^{+\infty} e^{2|\omega| T}|X(i \omega)|^{2} d \omega=\sum_{k=0}^{\infty} \frac{(2 T)^{k}}{k !} \int_{-\infty}^{+\infty}|\omega|^{k}|X(i \omega)|^{2} d \omega \leq 2 \pi M \sum_{k=0}^{\infty} \frac{(2 T)^{k}}{k !} \frac{k !}{C^{k}}<+\infty .
$$

It follows that $x \in \mathcal{X}(2, T)$. By Theorem $1(\mathrm{i})$, the required predictability holds.

Proof of Proposition 3. It is known that $K_{\mathcal{G}}(i \omega)=c \sqrt{\pi v} \exp \left(-v \pi^{2} \omega^{2}\right)$. It follows that $e^{|\omega| T} X(i \omega) \in L_{2}(\mathbf{R}) \cap L_{1}(\mathbf{R})$ and $x \in \mathcal{X}(1) \cap \mathcal{X}(2)$. It follows also that condition (ii) in Theorem 1 is satisfied for class $\mathcal{V}_{\mathcal{G}}$ for any $T>0$.

Remark 3 The predictors introduced above are stable, since the corresponding transfer functions belong to $H^{2} \cap H^{\infty}$. In addition, these predictors are robust with respect to the deviations of the process that are small in the weighted norm generated by the definition of the space $\mathcal{X}(q)$.

Remark 4 Formally, the predictors described above require the past values of $x(s)$ for all $s \in(-\infty, t]$, but it is not too restrictive, since $\int_{-\infty}^{t} \widehat{k}(t-s) x(s) d s$ can be approximated by $\int_{-M}^{t} \widehat{k}(t-s) x(s) d s$ for large enough $M>0$. In addition, the corresponding transfer functions can be approximated by rational fraction polynomials, and more general kernels $k$ can be approximated by kernels from $\mathcal{K}(T)$. 


\section{References}

[1] Beutler F.G. (1966). Error-free recovery of signals from irregularly spaced samples. SIAM Review, 8(3), 328-335.

[2] Brown J.R., Jr.. (1969). Bounds for truncation error in sampling expansion of bandlimited signals. IEEE Transactions Inform. Theory 15, no. 4, 440-444.

[3] Dokuchaev, N.G. (2008). The predictability of band-limited, high-frequency, and mixed processes in the presence of ideal low-pass filters. Journal of Physics A: Mathematical and Theoretical 41 No 38, 382002 (7pp).

[4] Duren P. (1970) Theory of $H^{p}$-Spaces. Academic Press, New York.

[5] Higgins, J.R. (1996). Sampling Theory in Fourier and Signal Analysis. Oxford University Press, New York.

[6] Knab J.J. (1979). Interpolation of band-limited functions using the approximate prolate series. IEEE Transactions on Information Theory 25(6), 717-720.

[7] Lyman R.J, Edmonson W.W., McCullough S., and Rao M. (2000). The predictability of continuous-time, bandlimited processes. IEEE Transactions on Signal Processing 48, Iss. 2, 311-316.

[8] Lyman R.J and Edmonson W.W. (2001). Linear prediction of bandlimited processes with flat spectral densities. IEEE Transactions on Signal Processing 49, Iss. 7, 15641569.

[9] Marvasti F. (1986). Comments on "A note on the predictability of band-limited processes." Proceedings of the IEEE, 74(11), 1596.

[10] Papoulis A. (1985). A note on the predictability of band-limited processes. Proceedings of the IEEE, 73(8), 1332-1333.

[11] Slepian D. (1978). Prolate spheroidal wave functions, Fourier analysis, and uncertainty-V: The discrete case. Bell System Technical Journal, 57(5), 1371-1430.

[12] Vaidyanathan P.P. (1987). On predicting a band-limited signal based on past sample values. Proceedings of the IEEE, 75(8), 1125-1127.

[13] Wainstein L.A. and Zubakov V.D. (1962). Extraction of Signals from Noise. Englewood Cliffs, NJ: Prentice-Hall. 